\title{
Merlin: Computer-Aided Oligonucleotide Design for Large Scale Genome Engineering with MAGE (Supplementary Info)
}

A

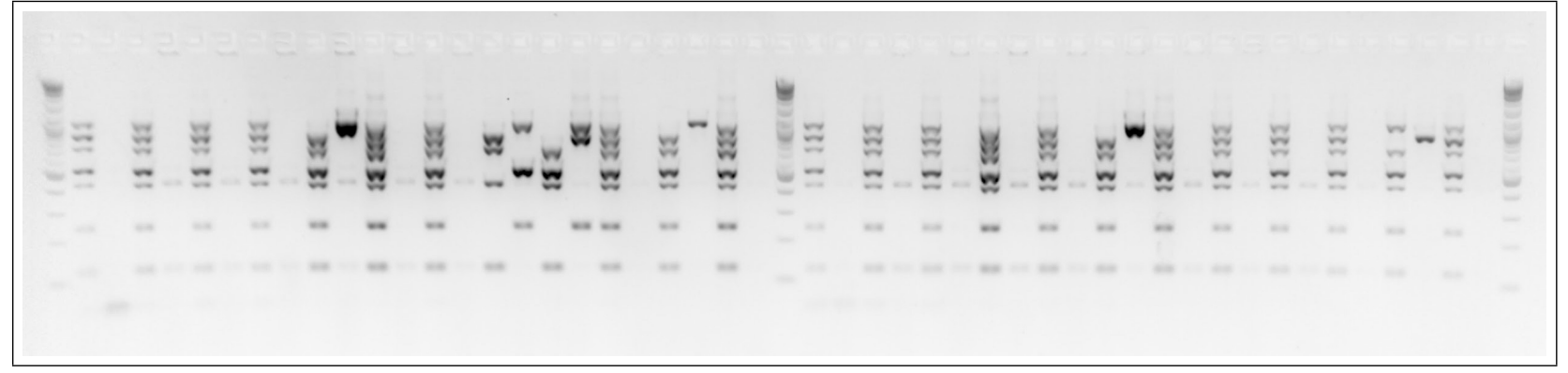

B

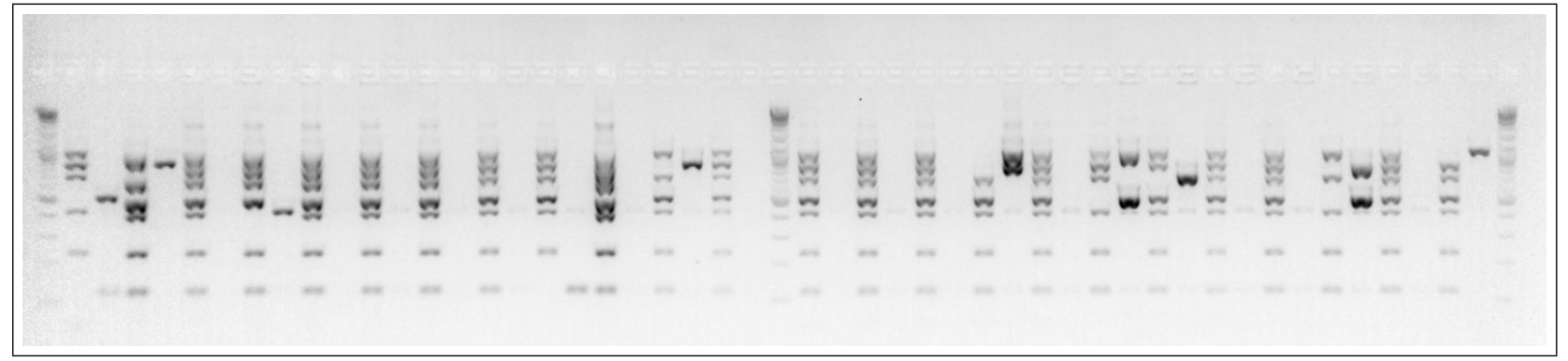

Figure 1: MASC-PCR gel used for primer validation. Images are of mASC-PCR reactions for clones. A: wells A1-B12. B: wells C1-D12. 
Table 1: Sanger Sequencing and PCR validation of Merlin Oligos. $0=$ wild type, $1=$ mutant.

\begin{tabular}{|c|c|c|c|c|c|c|c|c|c|c|c|c|c|c|}
\hline & \multicolumn{2}{|c|}{ AvrII01 } & \multicolumn{2}{|c|}{ AvrII02 } & \multicolumn{2}{|c|}{ AvrII03 } & \multicolumn{2}{|c|}{ AvrII04 } & \multicolumn{2}{|c|}{ AvrII05 } & \multicolumn{2}{|c|}{ AvrII06 } & \multicolumn{2}{|c|}{ AvrII07 } \\
\hline Well & PCR & Seq & PCR & Seq & PCR & Seq & PCR & Seq & PCR & Seq & PCR & Seq & PCR & Seq \\
\hline A1 & 0 & 0 & 0 & 0 & 0 & 0 & 0 & 0 & 0 & 0 & 0 & 0 & 0 & 0 \\
\hline A2 & 0 & 0 & 0 & 0 & 0 & 0 & 0 & 0 & 0 & 0 & 0 & 0 & 0 & 0 \\
\hline A3 & 0 & 0 & 0 & 0 & 0 & 0 & 0 & 0 & 0 & 0 & 0 & 0 & 0 & 0 \\
\hline A4 & 0 & 0 & 0 & 0 & 0 & 0 & 0 & 0 & 0 & 0 & 0 & 0 & 0 & 0 \\
\hline A5 & 0 & 0 & 0 & 0 & 0 & 0 & 0 & 0 & 1 & 1 & 0 & 0 & 0 & 0 \\
\hline A6 & 0 & 0 & 0 & 0 & 0 & 0 & 0 & 0 & 0 & 0 & 0 & 0 & 0 & 0 \\
\hline A7 & 0 & 0 & 0 & 0 & 0 & 0 & 0 & 0 & 0 & 0 & 0 & 0 & 0 & 0 \\
\hline A8 & 1 & 1 & 0 & 0 & 0 & 0 & 0 & 0 & 1 & 1 & 1 & 1 & 0 & 0 \\
\hline A9 & 1 & 1 & 0 & 0 & 0 & 0 & 1 & 1 & 1 & 1 & 0 & 0 & 0 & 0 \\
\hline A10 & 0 & 0 & 0 & 0 & 0 & 0 & 0 & 0 & 0 & 0 & 0 & 0 & 0 & 0 \\
\hline A11 & 0 & 0 & 0 & 0 & 0 & 0 & 0 & 0 & 1 & 1 & 0 & 0 & 0 & 0 \\
\hline A12 & 0 & 0 & 0 & 0 & 0 & 0 & 0 & 0 & 0 & 0 & 0 & 0 & 0 & 0 \\
\hline B1 & 0 & 0 & 0 & 0 & 0 & 0 & 0 & 0 & 0 & 0 & 0 & 0 & 0 & 0 \\
\hline B2 & 0 & 0 & 0 & 0 & 0 & 0 & 0 & 0 & 0 & 0 & 0 & 0 & 0 & 0 \\
\hline B3 & 0 & 0 & 0 & 0 & 0 & 0 & 0 & 0 & 0 & 0 & 0 & 0 & 0 & 0 \\
\hline B4 & 0 & 0 & 0 & 0 & 0 & 0 & 0 & 0 & 0 & 0 & 0 & 0 & 0 & 0 \\
\hline B5 & 0 & 0 & 0 & 0 & 0 & 0 & 0 & 0 & 0 & 0 & 0 & 0 & 0 & 0 \\
\hline B6 & 0 & 0 & 0 & 0 & 0 & 0 & 0 & 0 & 1 & 1 & 0 & 0 & 0 & 0 \\
\hline B7 & 0 & 0 & 0 & 0 & 0 & 0 & 0 & 0 & 0 & 0 & 0 & 0 & 0 & 0 \\
\hline B8 & 0 & 0 & 0 & 0 & 0 & 0 & 0 & 0 & 0 & 0 & 0 & 0 & 0 & 0 \\
\hline B9 & 0 & 0 & 0 & 0 & 0 & 0 & 0 & 0 & 0 & 0 & 0 & 0 & 0 & 0 \\
\hline $\mathrm{B} 10$ & 0 & 0 & 0 & 0 & 0 & 0 & 0 & 0 & 0 & 0 & 0 & 0 & 0 & 0 \\
\hline B11 & 0 & 0 & 0 & 0 & 0 & 0 & 1 & 1 & 0 & 0 & 0 & 0 & 0 & 0 \\
\hline B12 & 0 & 0 & 0 & 0 & 0 & 0 & 0 & 0 & 0 & 0 & 0 & 0 & 0 & 0 \\
\hline C1 & 0 & 0 & 0 & 0 & 0 & 0 & 0 & 0 & 0 & 0 & 1 & 1 & 1 & 1 \\
\hline $\mathrm{C} 2$ & 0 & 0 & 0 & 0 & 0 & 0 & 1 & 1 & 0 & 0 & 0 & 0 & 0 & 0 \\
\hline C3 & 0 & 0 & 0 & 0 & 0 & 0 & 0 & 0 & 0 & 0 & 0 & 0 & 0 & 0 \\
\hline $\mathrm{C} 4$ & 0 & 0 & 1 & 1 & 0 & 0 & 0 & 0 & 0 & 0 & 0 & 0 & 0 & 0 \\
\hline C5 & 0 & 0 & 0 & 0 & 0 & 0 & 0 & 0 & 0 & 0 & 0 & 0 & 0 & 0 \\
\hline C6 & 0 & 0 & 0 & 0 & 0 & 0 & 0 & 0 & 0 & 0 & 0 & 0 & 0 & 0 \\
\hline C7 & 0 & 0 & 0 & 0 & 0 & 0 & 0 & 0 & 0 & 0 & 0 & 0 & 0 & 0 \\
\hline C8 & 0 & 0 & 0 & 0 & 0 & 0 & 0 & 0 & 0 & 0 & 0 & 0 & 0 & 0 \\
\hline C9 & 0 & 0 & 0 & 0 & 0 & 0 & 0 & 0 & 0 & 0 & 0 & 0 & 1 & 1 \\
\hline C10 & 0 & 0 & 0 & 0 & 0 & 0 & 0 & 0 & 0 & 0 & 0 & 0 & 0 & 0 \\
\hline C11 & 0 & 0 & 0 & 0 & 0 & 0 & 1 & 1 & 0 & 0 & 0 & 0 & 0 & 0 \\
\hline C12 & 0 & 0 & 0 & 0 & 0 & 0 & 0 & 0 & 0 & 0 & 0 & 0 & 0 & 0 \\
\hline D1 & 0 & 0 & 0 & 0 & 0 & 0 & 0 & 0 & 0 & 0 & 0 & 0 & 0 & 0 \\
\hline D2 & 0 & 0 & 0 & 0 & 0 & 0 & 0 & 0 & 0 & 0 & 0 & 0 & 0 & 0 \\
\hline D3 & 0 & 0 & 0 & 0 & 0 & 0 & 0 & 0 & 0 & 0 & 0 & 0 & 0 & 0 \\
\hline $\mathrm{D} 4$ & 0 & 0 & 0 & 0 & 0 & 0 & 1 & 1 & 1 & 1 & 0 & 0 & 0 & 0 \\
\hline D5 & 0 & 0 & 0 & 0 & 0 & 0 & 0 & 0 & 0 & 0 & 0 & 0 & 0 & 0 \\
\hline D6 & 0 & 0 & 0 & 0 & 0 & 0 & 0 & 0 & 1 & 1 & 1 & 1 & 0 & 0 \\
\hline D7 & 0 & 0 & 0 & 0 & 1 & 1 & 0 & 0 & 0 & 0 & 0 & 0 & 0 & 0 \\
\hline D8 & 0 & 0 & 0 & 0 & 0 & 0 & 0 & 0 & 0 & 0 & 0 & 0 & 0 & 0 \\
\hline D9 & 0 & 0 & 0 & 0 & 0 & 0 & 0 & 0 & 0 & 0 & 0 & 0 & 0 & 0 \\
\hline D10 & 0 & 0 & 0 & 0 & 0 & 0 & 1 & 1 & 0 & 0 & 1 & 1 & 0 & 0 \\
\hline D11 & 0 & 0 & 0 & 0 & 0 & 0 & 0 & 0 & 0 & 0 & 0 & 0 & 0 & 0 \\
\hline D12 & 0 & 0 & 0 & 0 & 0 & 0 & 0 & 0 & 1 & 1 & 0 & 0 & 0 & 0 \\
\hline Sum & 2 & 2 & 1 & 1 & 1 & 1 & 6 & 6 & 8 & 8 & 4 & 4 & 2 & 2 \\
\hline
\end{tabular}




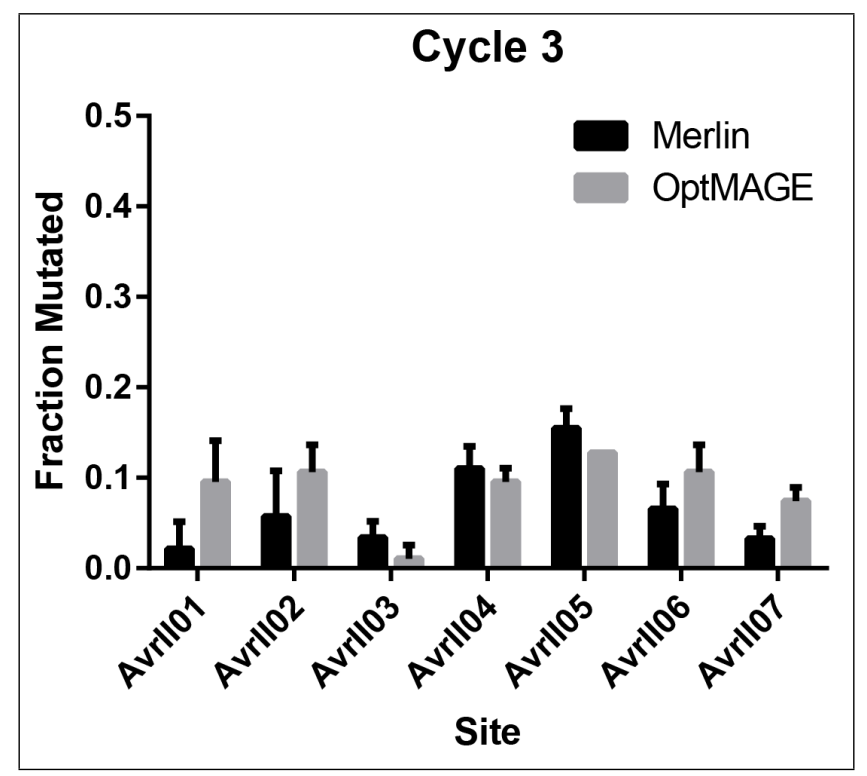

Figure 2: Diversity across all loci after 3 MAGE cycles. Error bars are the range of values for two separate MAGEd populations. $\mathrm{n}=47$.
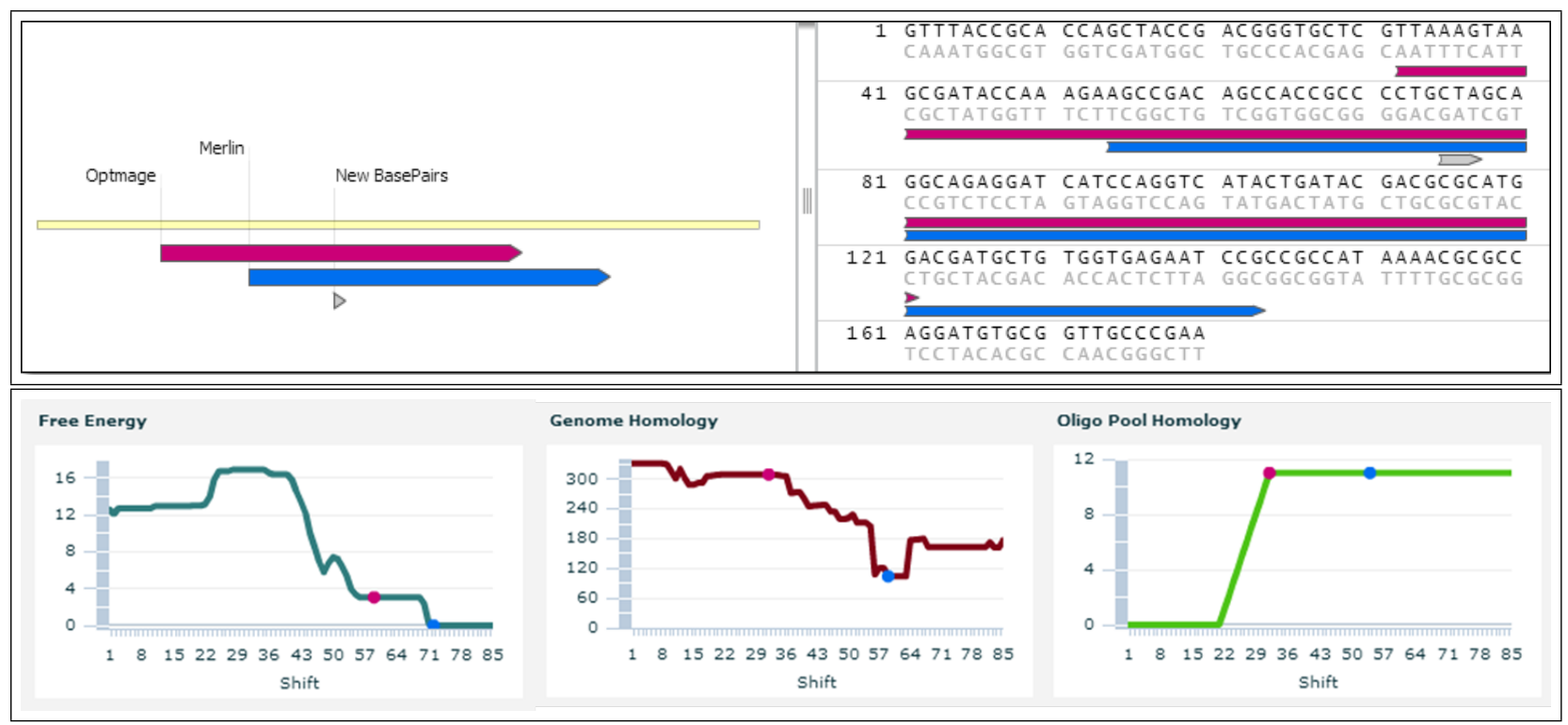

Figure 3: Example graphical output of the oligo optimization process, comparing oligos created by Merlin (blue) and OptMAGE (red). Top: Two alternate views of the location of the oligo spans and the targeted position, in this case a TAG insertion. Bottom: Plots of the (left to right) $\Delta$ G score, genome-wide BLAST score, and inter-oligo BLAST score for each valid oligo start position. The positions used and the resulting scores for the Merlin and OptMAGE oligos are depicted in blue and red, respectively. 


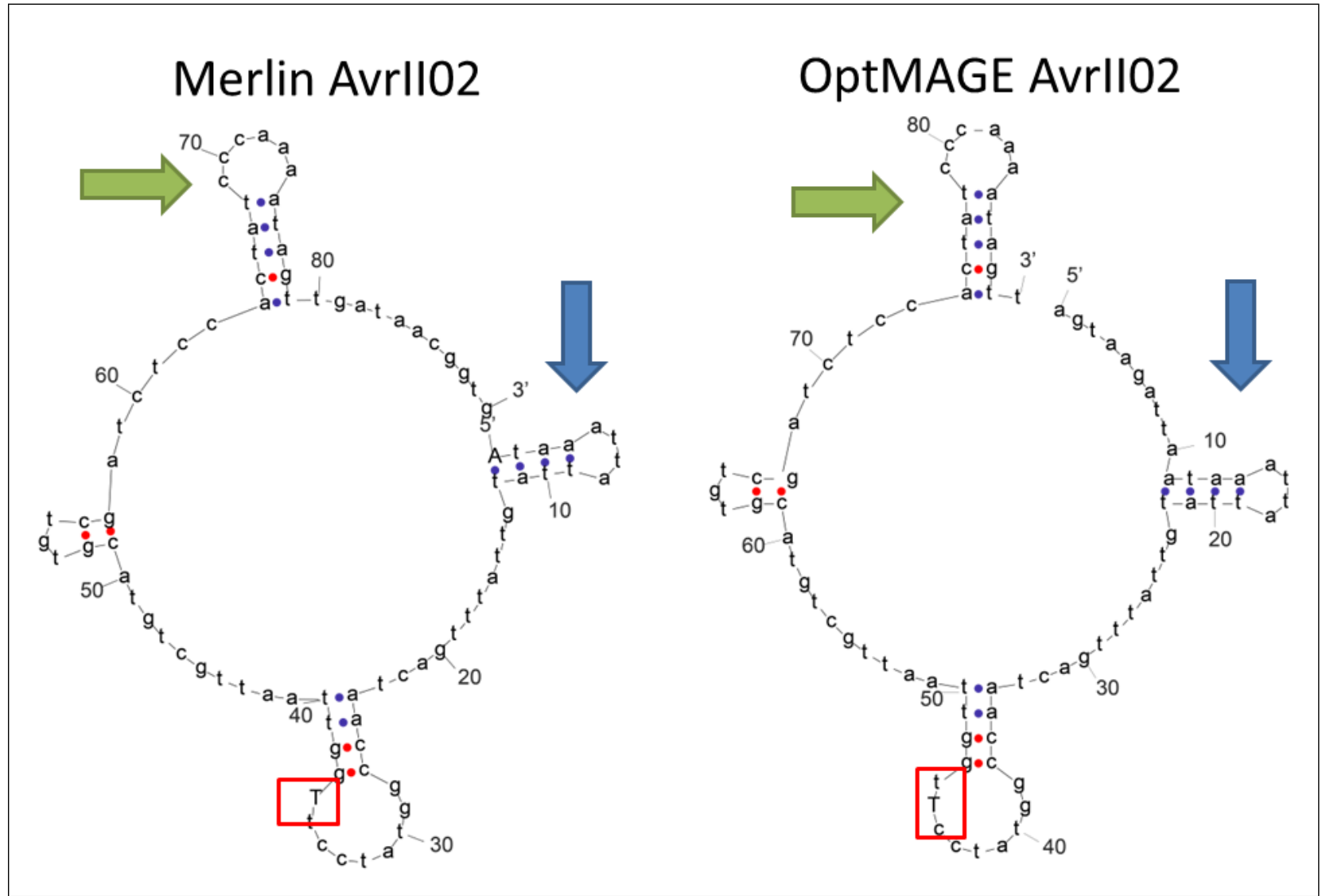

Figure 4: Comparison of predicted structures of oligos targeting AvrII02. The blue arrow indicates the $5^{\prime}$ hairpin, the green arrow indicates the $3^{\prime}$ hairpin, and the red box indicates the site of mutation. Structure prediction performed with mFold. 
Table 2: Sequence and predicted structure in dot-bracket notation of oligos generated by Merlin and OptMAGE. Structure prediction performed with mFold.

\begin{tabular}{|c|c|c|c|}
\hline Name & $\mathrm{dG}$ & Sequence & Structure \\
\hline avrII01-Mer & -28 & $\begin{array}{l}\text { tcatcacgtt tatcggtcgt cccggcaacg } \\
\text { cggttaagag attcttgatc tgcccagtca } \\
\text { taacgaccgc ctagGgtgac cagcacttta }\end{array}$ & 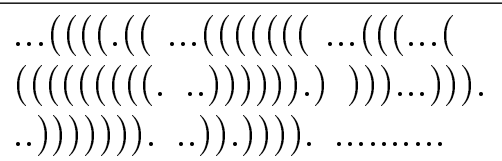 \\
\hline avrII02-Mer & -11.3 & $\begin{array}{l}\text { ataaattatt atgttatttg actaaccggt } \\
\text { atcctTggtt aattgctgta cgtgtcgatc } \\
\text { tccactatcc caaaatagtt gataacggtg }\end{array}$ & 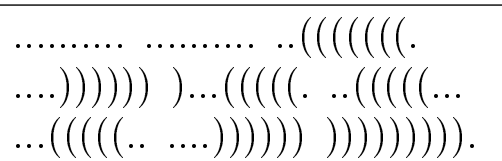 \\
\hline avrII03-Mer & -16.3 & $\begin{array}{l}\text { tgtcactgtt gggagttgta atgcaatata } \\
\text { atacgataat aattccctTg gttaaacagt } \\
\text { ctcagtacta aagatgtatg tgaaggggcc }\end{array}$ & $\begin{array}{l}(((.((((. . \quad(((((((((.)((\ldots \ldots \ldots . . \\
\ldots . .)) .))))))))) .)))) \ldots)))(( \\
(((. .((((. . \ldots \ldots \ldots .))) . . .)))))\end{array}$ \\
\hline avrII04-Mer & -10.7 & $\begin{array}{l}\text { ctgaagtaag aattatTcta ggggatgctg } \\
\text { tagtggaagt agcaaaacag ggaagaccta } \\
\text { tcaataccag aacattgctt gattacattg }\end{array}$ & 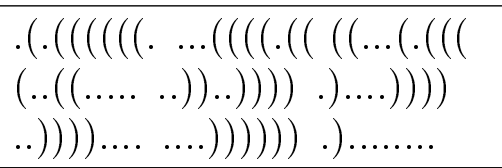 \\
\hline avrII05-Mer & -10.7 & $\begin{array}{l}\text { ctgaagtaag aattatTcta ggggatgctg } \\
\text { tagtggaagt agcaaaacag ggaagaccta } \\
\text { tcaataccag aacattgctt gattacattg }\end{array}$ & $\begin{array}{l}.(.((((((\ldots \ldots((((.)((((\ldots) .((() \\
(. .((\ldots \ldots \ldots)) . . .)))) \quad .) \ldots . . .)))) \\
. .)))) \ldots \ldots \ldots . \ldots))))) . . \ldots \ldots \ldots\end{array}$ \\
\hline avrII06-Mer & -12.7 & $\begin{array}{l}\text { gaagcattaa atgacttaaa cagttatacc } \\
\text { aatgctaata gtgttacttc cctaGgtgca } \\
\text { agactggcaa tgtacgaaat tggtttaaat }\end{array}$ & 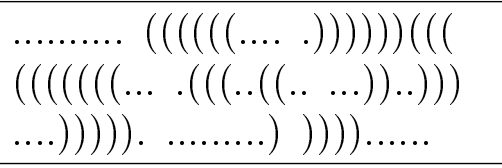 \\
\hline avrII07-Mer & -19.6 & $\begin{array}{l}\text { ccaaagggcc agcctttgtc gagcggtgta } \\
\text { ccgtgcttta atctgcaatc tttatcaaca } \\
\text { caagcccata ttccTtaggt attacgctgg }\end{array}$ & 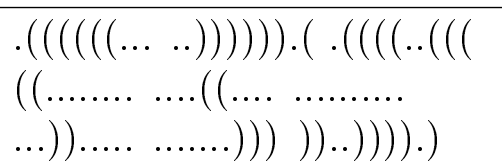 \\
\hline avrII01-Opt & -12.4 & $\begin{array}{l}\text { gcggttaaga gattcttgat ctgcccagtc } \\
\text { ataacgaccg cctagCgtga ccagcacttt } \\
\text { atcccactgc gcctgatcct gaacataaac }\end{array}$ & $\begin{array}{l}((((((((((\ldots))))))) \cdot)))) \ldots((( \\
\ldots \ldots)))(((\ldots))) \cdot((\cdot((((.(\ldots \\
\ldots \ldots . . .) .) .))) .)) . . \ldots \ldots \ldots . .\end{array}$ \\
\hline avrII02-Opt & -8.8 & $\begin{array}{l}\text { agtaagatta ataaattatt atgttatttg } \\
\text { actaaccggt atccTtggtt aattgctgta } \\
\text { cgtgtcgatc tccactatcc caaaatagtt }\end{array}$ & 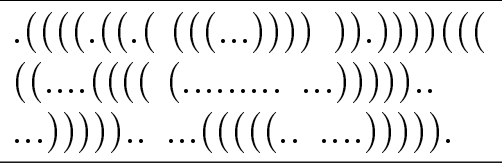 \\
\hline avrII03-Opt & -15.5 & $\begin{array}{l}\text { cactgttggg agttgtaatg caatataata } \\
\text { cgataataat tccctTggtt aaacagtctc } \\
\text { agtactaaag atgtatgtga aggggccgcg }\end{array}$ & 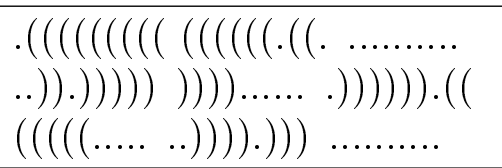 \\
\hline avrII04-Opt & -24.7 & $\begin{array}{l}\text { caccctaaag tggttcctg cgcagcttcc } \\
\text { tgaagtaaga attatTctag gggatgctgt } \\
\text { agtggaagta gcaaaacagg gaagacctat }\end{array}$ & 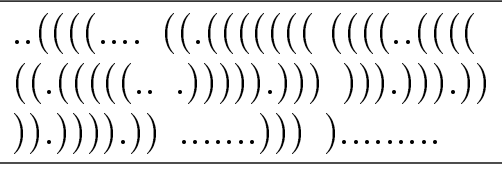 \\
\hline avrII05-Opt & -24.7 & $\begin{array}{l}\text { caccctaaag tggttccetg cgcagcttcc } \\
\text { tgaagtaaga attatTctag gggatgctgt } \\
\text { agtggaagta gcaaaacagg gaagacctat }\end{array}$ & $\begin{array}{l}. .((((\ldots .((.((((((((((((. .)(((() \\
((.(((((\ldots . .))))) .))))))) .))) .)) \\
)) .)))) .)) \quad \ldots \ldots . . .))))) \ldots \ldots \ldots . . \\
\end{array}$ \\
\hline avrII06-Opt & -10.8 & $\begin{array}{l}\text { aatgacttaa acagttatac caatgctaat } \\
\text { agtgttactt ccctaCgtgc aagactggca } \\
\text { atgtacgaaa ttggtttaaa tatattcata }\end{array}$ & 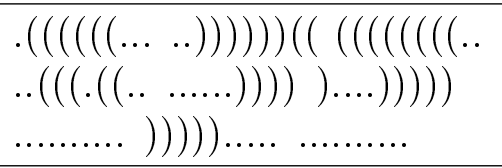 \\
\hline avrII07-Opt & -10.7 & $\begin{array}{l}\text { accgtgcttt aatctgcaat gtttatcaac } \\
\text { acaagcccat attccTtagg tattacgctg } \\
\text { gaagatatgc gcctgactt taagaaatgc }\end{array}$ & $\begin{array}{l}\ldots . .(((\ldots \ldots \ldots))) \cdot((((\ldots \ldots .)( \\
(\ldots((.((((((((\ldots . . \ldots \ldots) \ldots) \\
)) . .))))) . .)) . . .)) \ldots . . .)))) \ldots \ldots\end{array}$ \\
\hline
\end{tabular}

